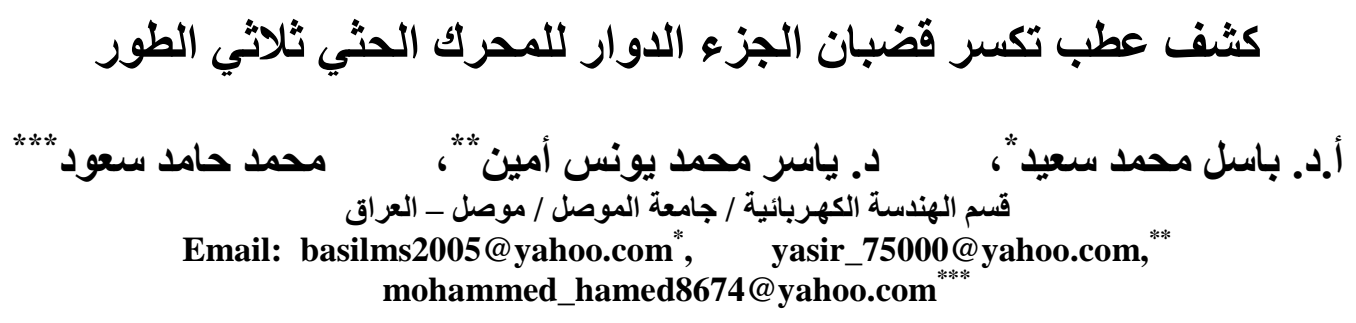

الخلاصة

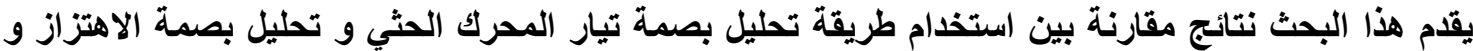

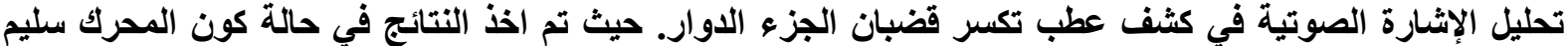

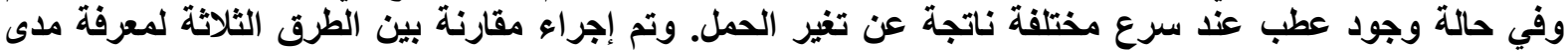

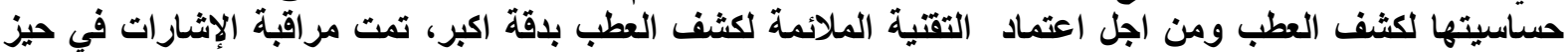

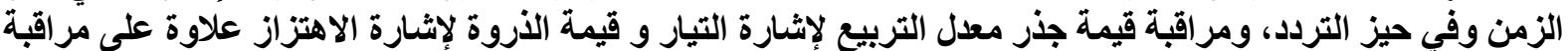

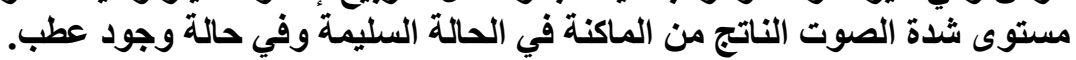

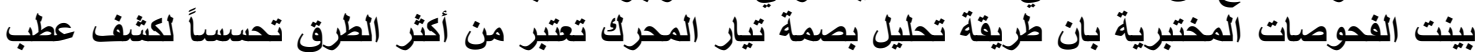

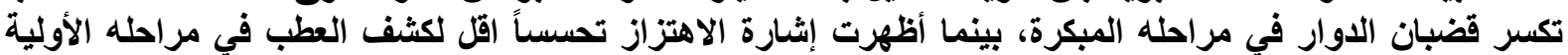

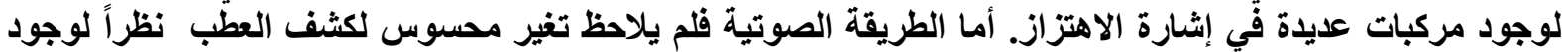
ضوضاء في بيئة القياس ولترددات فئرة العطب الواطئة. الكلمات المفتاحية : تكسر قضبان الجزء الدوار، تحليل بصمة التيار، تحليل الاهتزاز، الضوضاء الصوتي.

\title{
Broken Rotor Bars Fault Detection of The Three-phase Induction Motor
}

\author{
Prof. B. M. Saied
}

\author{
Dr. Y. M.Y. Ameen.
}

\begin{abstract}
This paper presents results of the comparison between the use of induction motor current signature, vibration signature, and acoustic signal analysis methods to detect broken rotor bars fault. The results have taken at healthy and faulty conditions at different speeds due to change of load. It is a comparison between three ways to determine the sensitivity for detecting faults and to adopt the best technology in detecting damages in order to increase the reliability of the system. Signals are been analyzed in time and frequency domains. The value of the root mean square of the current signal, the peak value of vibration signal, and the sound pressure level of the machine sound have monitored for healthy and faulty conditions.

The laboratory tests show that the results of analyzing the current signature is considered one of the most sensitive to detect the rotor broken bar faults in its early stages، while the vibration signal method is less sensitive to detect the fault in the initial stages because of the presence of other components in the vibration signal. But the acoustic method is not reliable method for detecting this type of the faults. This is due to the presence of noise in the environment and fault low order frequencies.
\end{abstract}

Keywords: Broken rotor bars, Current signature, Vibration Analysis, Acoustic Noise. 
تستخدم المكائن الحثية ثلاثية الطور بشكل واسع في المجال الصناعي حيث تمثل نسبتها ما يقارب

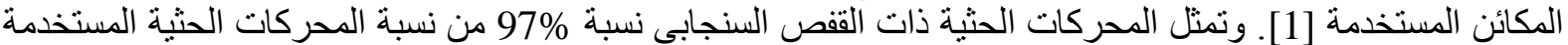

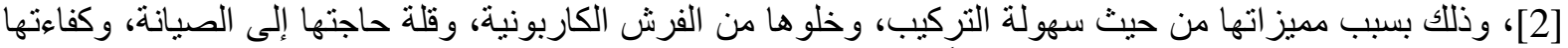

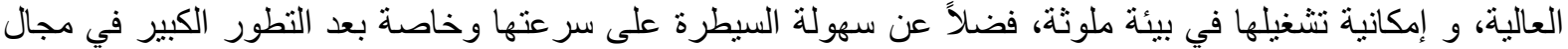
الكترونيات القدرة و صناعة المسوقات (Drives).

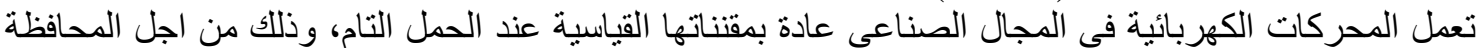

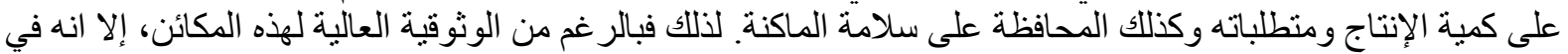

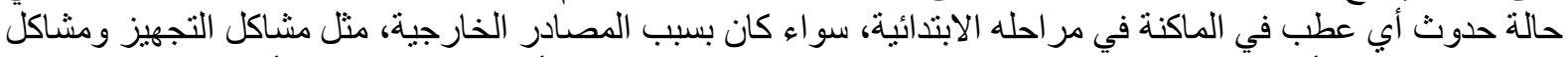

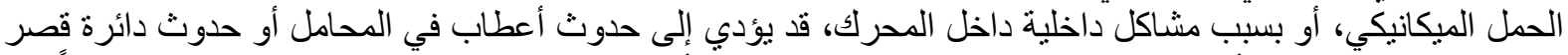

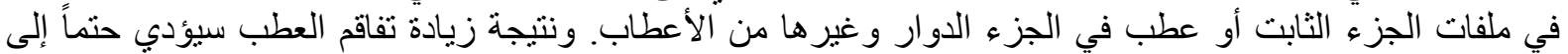

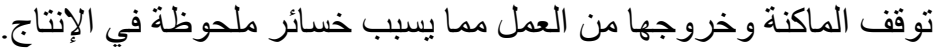

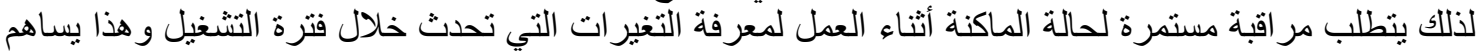

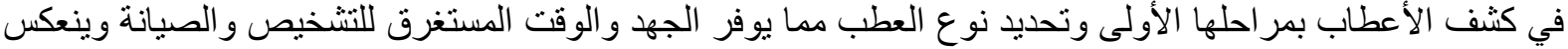

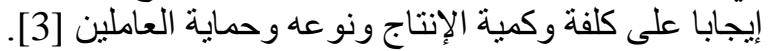

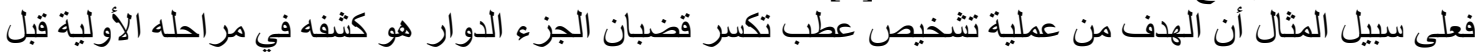

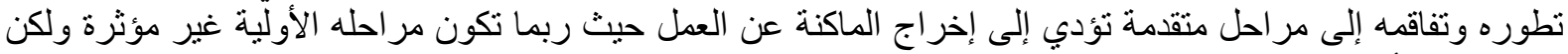

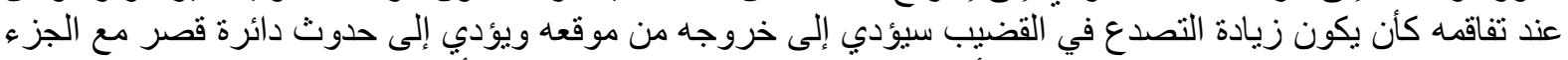

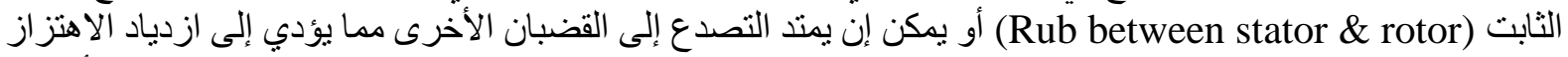

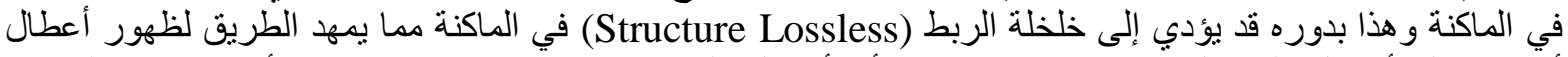
أخرى مثل أعطال المحامل (Bearing Fault) أو أعطال المعشق (Coupling Defect) أو صندوق الطريط التعشيق

.(Gearbox Defect)

\section{(Broken Rotor Bars Fault)}

2- 2 عطب تكسر قضبان الجزء الدوار

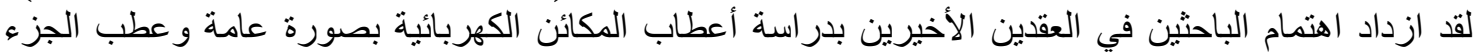

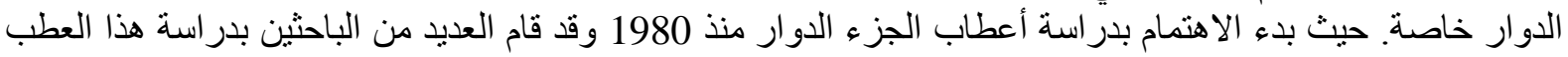

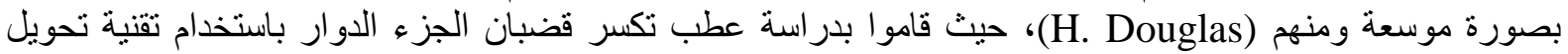
فورير السريع (FFT) وتحويل المويجة المقطع (Discrete Wavelet Transform) خلال الفترة العابرة التي تحصل فئل عند تغير الحمل [4]. وقام (K. Abbasadeh)، بدراسة العطب باستخدام تقنية تحويل المويجة Wavelet) (5) وباستخدام تقنية تحويل حزمة المويجة (Wavelet Packet Transform (5).

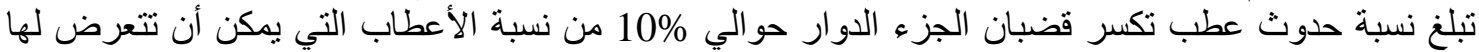

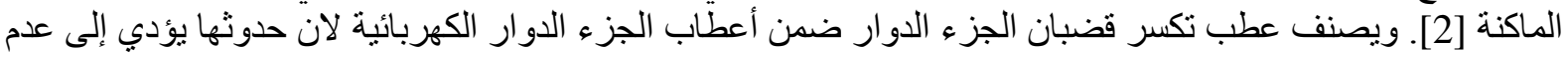

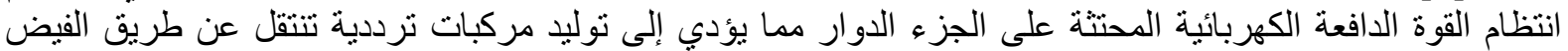

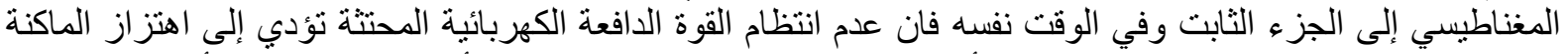

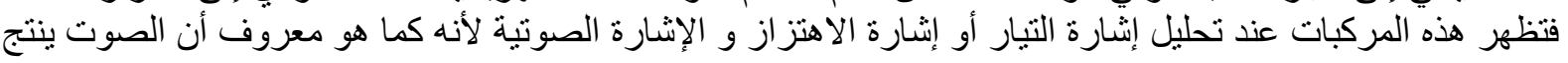
من اهنز از جسم معين. يحدث عطب تكسر قضبان الجزء الدوار نتيجة تعرض الماكنة إلى إجهادات تؤدي إلى حدوث العطب [6] ويمكن تلخيص هذه العوامل إلى :-

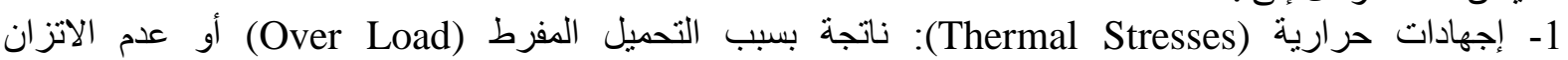
(Unbalance) 2- إجهادات مغناطيسية (Magnetic Stresses): ناتجة عن عدم اتزان قوة الجذب الكهرومغناطيسية (UMP) (U) التي لتنتج بسبب عدم اتزان الفجوة الهوائية (Air Gap Eccentricity). 3- إجهادات ميكانيكية (Mechanical Stresses) نانجة (Bearing Fatigue) (أنجة عن إعياء في المحامل عدم محاذاة محور الدوران (Shaft Misalignment). 4- إجهادات حركية (Dynamic stresses): ناتجة بسبب عدم النتام قورة الجذب المركزية (Centrifugal Force)

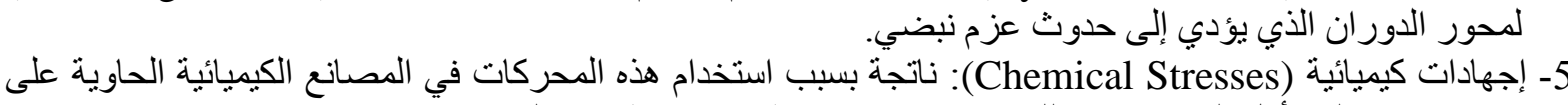

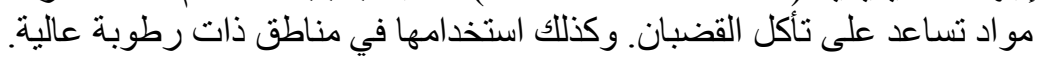
6- إجهادات ناتجة بسبب عيوب تصنيعية (Manufacture Defects). 
علاوة على هذه العوامل فان التشغيل المستمر للمحرك بطريقة مبانشرة بدون وجود مسوق قد بؤن بؤدي إلى حدوث تصدع

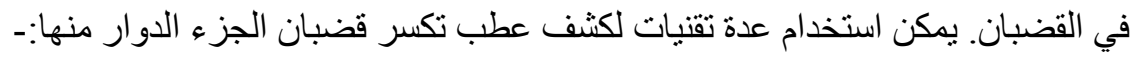

1- تقنية مر اقبة الفيض الكهرومغناطيسي (Electromagnetic Flux Monitoring Technique).

2- تقنية مر اقبة الاهتزاز (Vibration Monitoring Technique).

3- التقنية الصوتية (Acoustic Emission Monitoring Technique)

4- تقنية مر اقبة تيار الماكنة (Current Monitoring Technique)

5- تقية مر اقبة فولتية محور الدوران (Shaft Voltage Monitoring Technique)

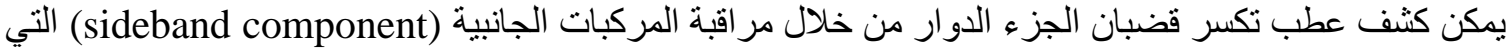

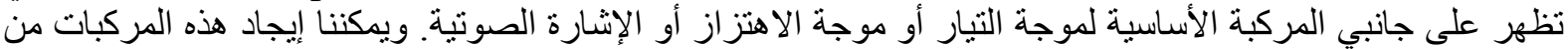
خلال مر اقبة الإثار ات الناتجة من الماكنة و عن طريق العلاقة الاتية [5]،؛[4]، [2]:-

$\mathrm{F}_{\text {brb_c }}=\mathrm{f}_{\mathrm{s}}(1 \pm 2 \mathrm{~S}) \quad\{$ In current signal $\}$

$\mathrm{F}_{\text {brb_V\&A }}=\mathrm{F}_{\mathrm{sh}}(1 \pm 2 \mathrm{~S}) \quad\{$ In Vibration and Acoustic signals $\}$

حيث تمثل :Frb_c تردد المركبات الجانبية الناتجة من عطب تكسر قضبان الجزء الدوار في إثارة التبار.

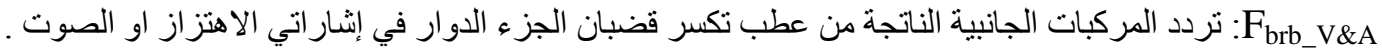

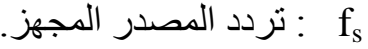

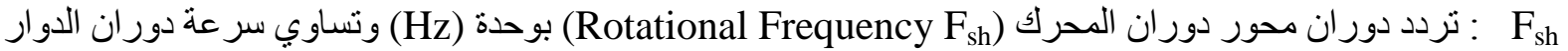

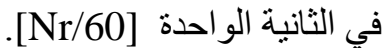

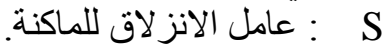

\section{3- منظومة الكثف والتشخيص}

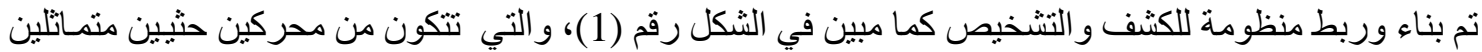

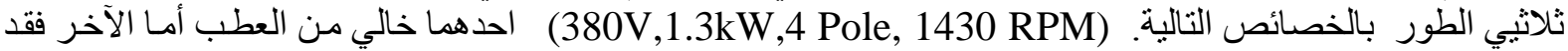
تم ثقب ثلاثة قضبان متجاورة في الجزء الدوار وذلك من اجل محاكاة عطب تكسر قضبان الجزء الدوار [7].

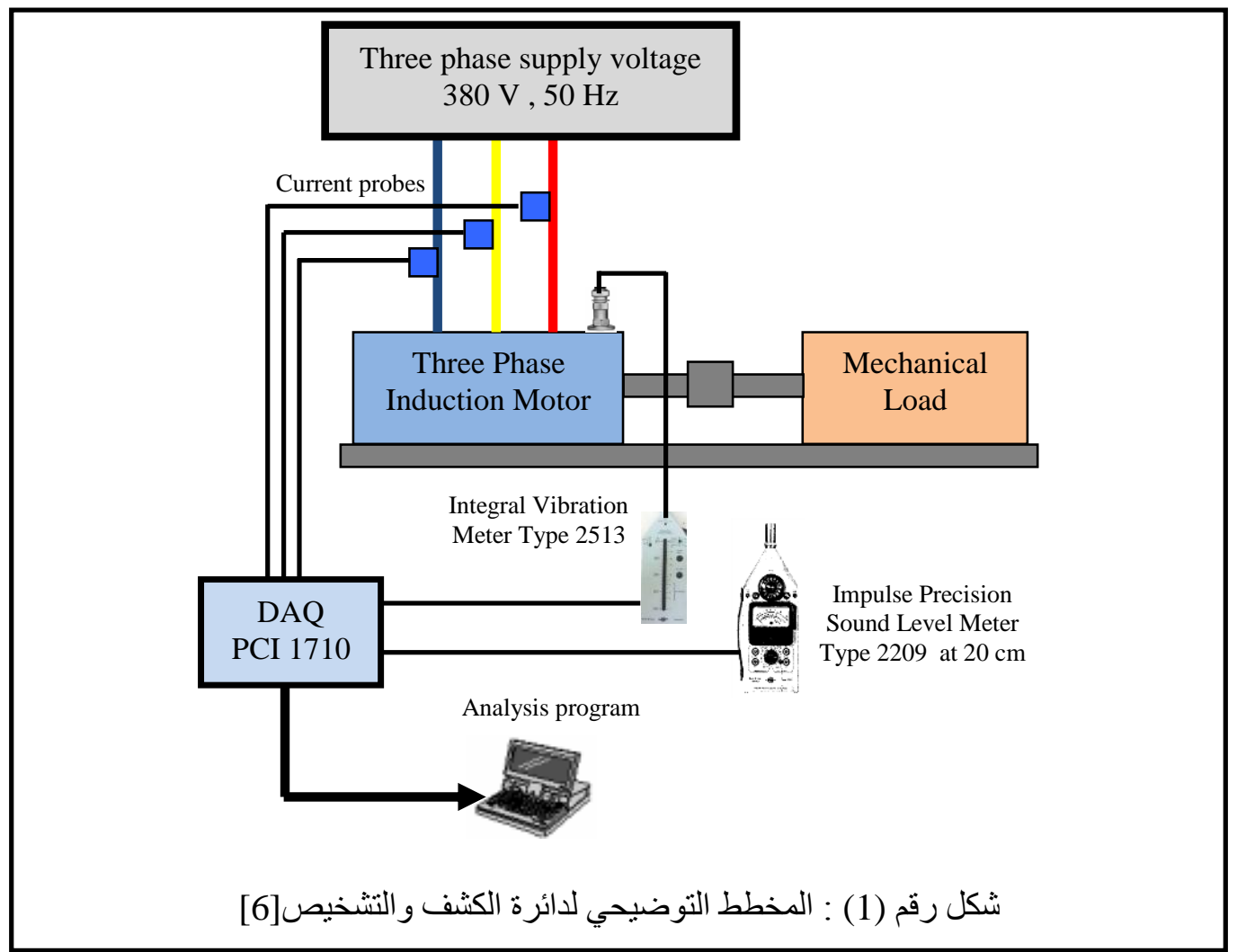




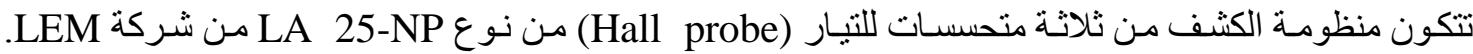

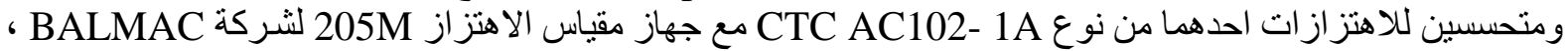

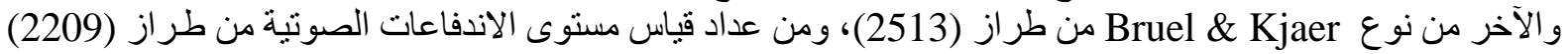

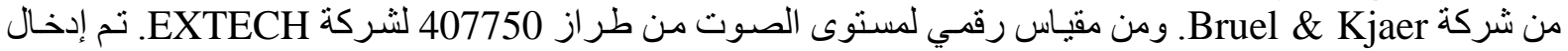

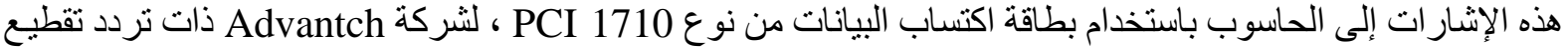

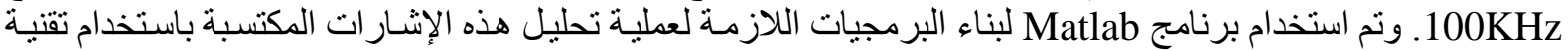
تحويل فورير السريع (FFT) حيث تم إدخال الإشارات باستخدام تردد اخذ عينات قدره (Fs = 4096 Hz) وبعدد عينات

$$
\text { . }\left(\mathrm{N}_{\mathrm{sa}}=32768\right)
$$

4- النتائج والمناقشة

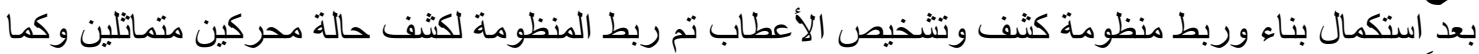

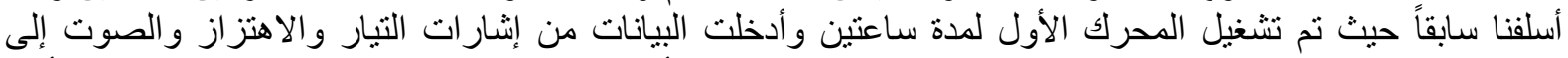

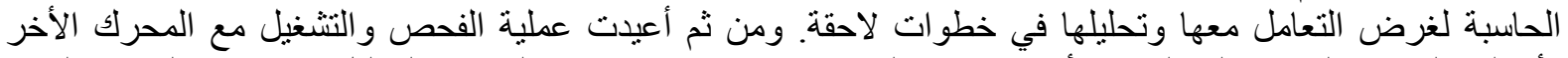

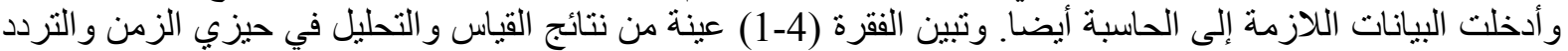
و التي تم الحصول عليها من حالات تشغيل مختلفة.

\section{(Time Domain Measurement Results)}

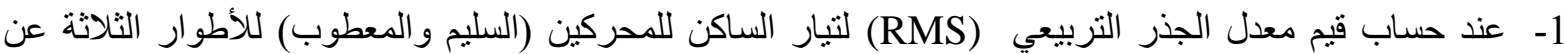

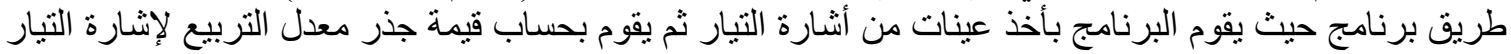

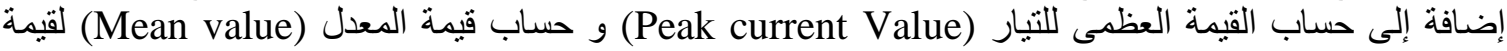

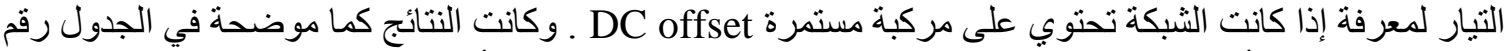

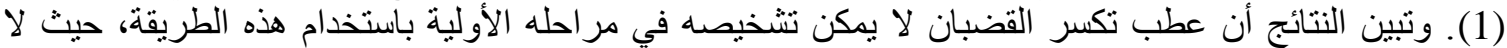

$$
\text { يؤدي إلى ارتفاع ملحوظ في قيمة التيار المسحوب. }
$$

جدول رقم (1) تيار ات الأطو ار الثثلاثة للمحركين السليم و المعطوب

\begin{tabular}{|c|c|c|c|c|c|c|c|}
\hline Load & \multirow{2}{*}{$\begin{array}{c}\text { Speed } \\
\end{array}$} & \multicolumn{3}{|c|}{ Healthy motor current } & \multicolumn{3}{c|}{ Faulty motor Current } \\
\cline { 3 - 8 } & $(\mathrm{RPM})$ & $\mathrm{I}_{\mathrm{R}}(\mathrm{A})$ & $\mathrm{I}_{\mathrm{S}}(\mathrm{A})$ & $\mathrm{I}_{\mathrm{T}}(\mathrm{A})$ & $\mathrm{I}_{\mathrm{R}}(\mathrm{A})$ & $\mathrm{I}_{\mathrm{S}}(\mathrm{A})$ & $\mathrm{I}_{\mathrm{T}}(\mathrm{A})$ \\
\hline N.L & 1490 & 1.73 & 1.72 & 1.74 & 1.73 & 1.72 & 1.73 \\
\hline H.L & 1450 & 2.41 & 2.45 & 2.44 & 2.43 & 2.44 & 2.44 \\
\hline F.L & 1430 & 3.15 & 3.08 & 3.13 & 3.11 & 3.14 & 3.16 \\
\hline
\end{tabular}

2- عند قياس مستوى الاهتز از للمحركين (السليم والمعطوب) عند الاتجاهات الثلاثة (عمودي، أفقي، محوري) كانت نتائج

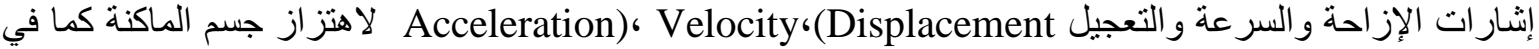
الجداول (2 و 3) على التو الي للاتجاهات الثناثة.

جدول رقم (2) مستوى اهتز از المحرك السليم

\begin{tabular}{|c|c|c|c|c|c|c|c|c|c|}
\hline L & \multicolumn{3}{|c|}{ Vertical Position } & \multicolumn{3}{c|}{ Horizontal Position } & \multicolumn{3}{c|}{ Axial Position } \\
\cline { 2 - 10 } $\begin{array}{c}\text { A } \\
\text { D }\end{array}$ & $\begin{array}{c}\text { Acc } \\
\left(\mathbf{m m} / \mathbf{s}^{2}\right)\end{array}$ & $\begin{array}{c}\text { Vel } \\
(\mathbf{m m} / \mathbf{s})\end{array}$ & $\begin{array}{c}\text { Dis } \\
(\mathbf{m m})\end{array}$ & $\begin{array}{c}\text { Acc } \\
\left(\mathbf{m m} / \mathbf{s}^{2}\right)\end{array}$ & $\begin{array}{c}\text { Vel } \\
(\mathbf{m m} / \mathbf{s})\end{array}$ & $\begin{array}{c}\text { Dis } \\
(\mathbf{m m})\end{array}$ & $\begin{array}{c}\text { Acc } \\
\left(\mathbf{m m} / \mathbf{s}^{2}\right)\end{array}$ & $\begin{array}{c}\text { Vel } \\
(\mathbf{m m} / \mathbf{s})\end{array}$ & $\begin{array}{c}\text { Dis } \\
(\mathbf{m m})\end{array}$ \\
\hline N.L & 0.22 & 0.95 & 6.81 & 0.16 & 0.89 & 9.8 & 0.06 & 0.66 & 4.35 \\
\hline H.L & 0.28 & 1.86 & 35.6 & 0.27 & 1.1 & 10.2 & 0.11 & 0.89 & 6.16 \\
\hline F.L & 0.34 & 1.67 & 31.5 & 0.23 & 1.07 & 7.6 & 0.12 & 0.85 & 6.35 \\
\hline
\end{tabular}


جدول رقم (3) مستوى اهتز از المحرك المعطوب الذي يحتوي على ثلاث قضبان مكسورة

\begin{tabular}{|c|c|c|c|c|c|c|c|c|c|}
\hline $\begin{array}{c}\mathrm{L} \\
\mathrm{O} \\
\mathrm{A} \\
\mathrm{D}\end{array}$ & \multicolumn{3}{|c|}{ Vertical Position } & \multicolumn{3}{c|}{ Horizontal Position } & \multicolumn{3}{c|}{ Axial Position } \\
\cline { 2 - 11 } & $\begin{array}{c}\text { Acc } \\
\left(\mathrm{mm} / \mathrm{s}^{2}\right)\end{array}$ & $\begin{array}{c}\text { Vel } \\
(\mathrm{mm} / \mathrm{s})\end{array}$ & $\begin{array}{c}\text { Dis } \\
(\mathrm{mm})\end{array}$ & $\begin{array}{c}\text { Acc } \\
\left(\mathrm{mm} / \mathrm{s}^{2}\right)\end{array}$ & $\begin{array}{c}\text { Vel } \\
(\mathrm{mm} / \mathrm{s})\end{array}$ & $\begin{array}{c}\text { Dis } \\
(\mathrm{mm})\end{array}$ & $\begin{array}{c}\text { Acc } \\
\left(\mathrm{mm} / \mathrm{s}^{2}\right)\end{array}$ & $\begin{array}{c}\text { Vel } \\
(\mathrm{mm} / \mathrm{s})\end{array}$ & $\begin{array}{c}\text { Dis } \\
(\mathrm{mm})\end{array}$ \\
\hline N.L & 0.21 & 1.46 & 16.23 & 0.24 & 0.86 & 8.28 & 0.11 & 0.74 & 3.24 \\
\hline H.L & 0.26 & 2.08 & 23.7 & 0.29 & 1.97 & 10.5 & 0.13 & 1.09 & 6.73 \\
\hline F.L & 0.29 & 1.96 & 22.6 & 0.45 & 1.93 & 9.25 & 0.12 & 1.23 & 6.75 \\
\hline
\end{tabular}

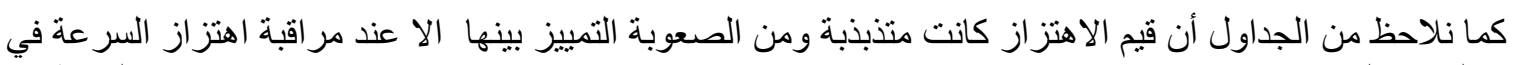

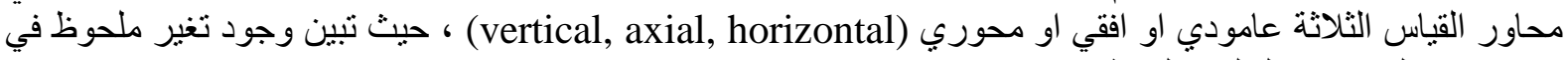

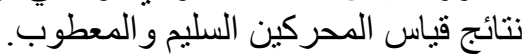
3- عند قياس مستوى شدة الصوت للدئ الدركين كانت النتائج كما مبينة في الجدول (4)، حيث تبين النتائج على عدم وجود زيادة ملحوظة في شدة صوت شند الماكنة مع حدوث العطب.

جدول رقم (4) مستوى شدة صوت المحرك السليم و المعطوب.

\begin{tabular}{|c|c|c|c|}
\hline Load & Speed (RPM) & Healthy motor noise (dB) & Faulty motor noise (dB) \\
\hline N.L & 1490 & 76.4 & 77.4 \\
\hline H.L & 1450 & 77.9 & 78.8 \\
\hline F.L & 1430 & 80.3 & 79.5 \\
\hline
\end{tabular}

2-4 نتائج تحليل الإشارات المكتسبة في حيز التردد:-

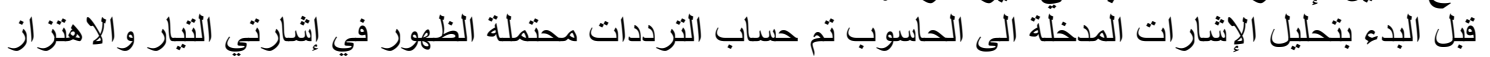

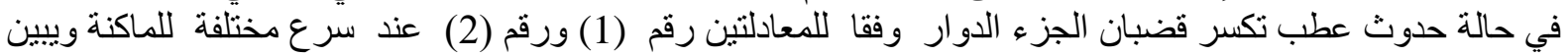

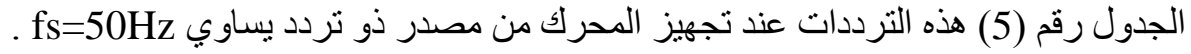

\begin{tabular}{|c|c|c|c|c|c|c|c|}
\hline \multirow{2}{*}{$\begin{array}{l}\text { Load } \\
\text { N.L }\end{array}$} & \multirow{2}{*}{$\begin{array}{c}\begin{array}{c}\text { Speed } \\
\text { (R.P.M) }\end{array} \\
1490 \\
\end{array}$} & \multirow{2}{*}{$\begin{array}{c}\mathrm{F}_{\mathrm{sh}}(\mathrm{Hz}) \\
24.83\end{array}$} & \multirow{2}{*}{$\begin{array}{c}\text { Slip factor } \\
\text { S } \\
0.67 \%\end{array}$} & \multicolumn{2}{|c|}{$\begin{array}{l}\text { Current sideband } \\
\text { freq. }(\mathrm{Hz}) \\
\mathrm{F}_{\text {brb_c }}\end{array}$} & \multicolumn{2}{|c|}{$\begin{array}{c}\text { Vibration \&Acostic } \\
\text { noise sideband (Hz) } \\
\text { F }_{\text {brb_V\&A }}\end{array}$} \\
\hline & & & & 50.67 & 49.33 & 25.163 & 24.497 \\
\hline H.L & 1450 & 24.167 & $3.33 \%$ & 53.33 & 46.67 & 25.776 & 22.556 \\
\hline F.L & 1430 & 23.83 & $4.67 \%$ & 54.67 & 45.33 & 26.055 & 21.604 \\
\hline
\end{tabular}

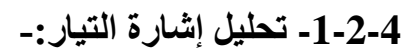

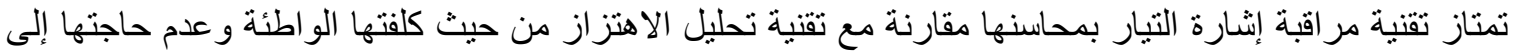

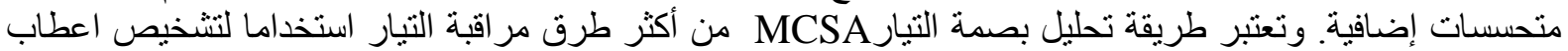

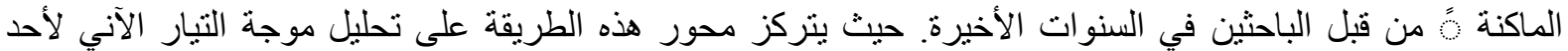

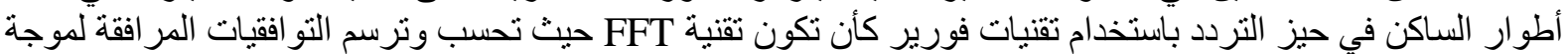

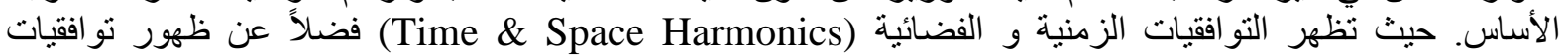
الاعطاب المبينة في العلاقة (1).

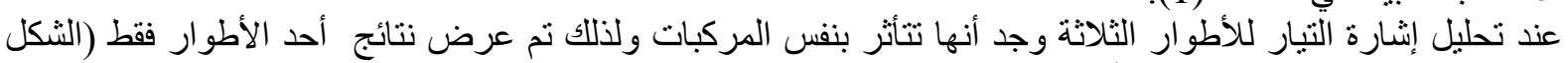

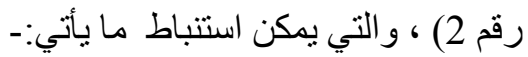
• عند تحليل إنثارة التيار في حالة التلاحمل لا يوجد ما يثير إلى وجود عطب نكسر القضبان كما موضح في الثكل رقم (2-A)

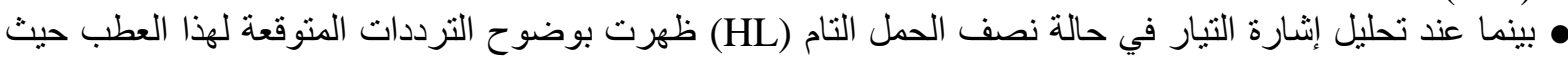
نلاحظ المركبات الظاهرة على جانبي المركبة الأساسية حيث كانت قيمة المركبة الجانبية اليسرى (left sideband) 


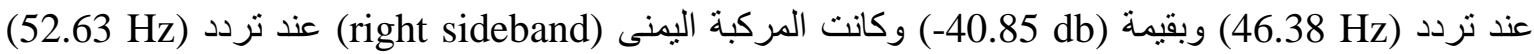

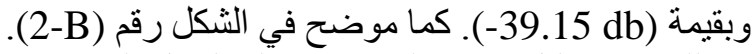

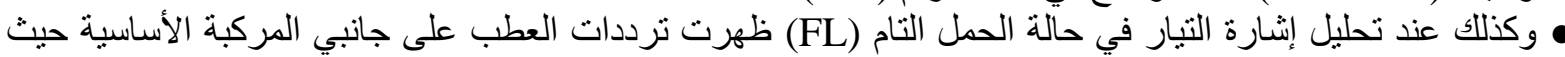

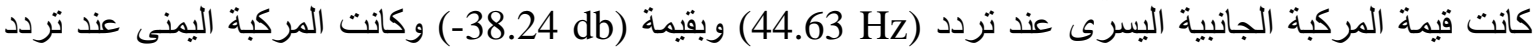

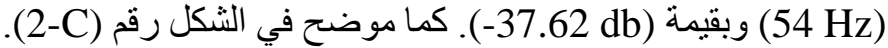

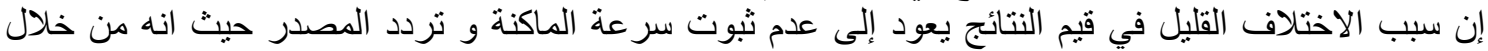

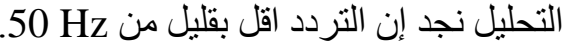

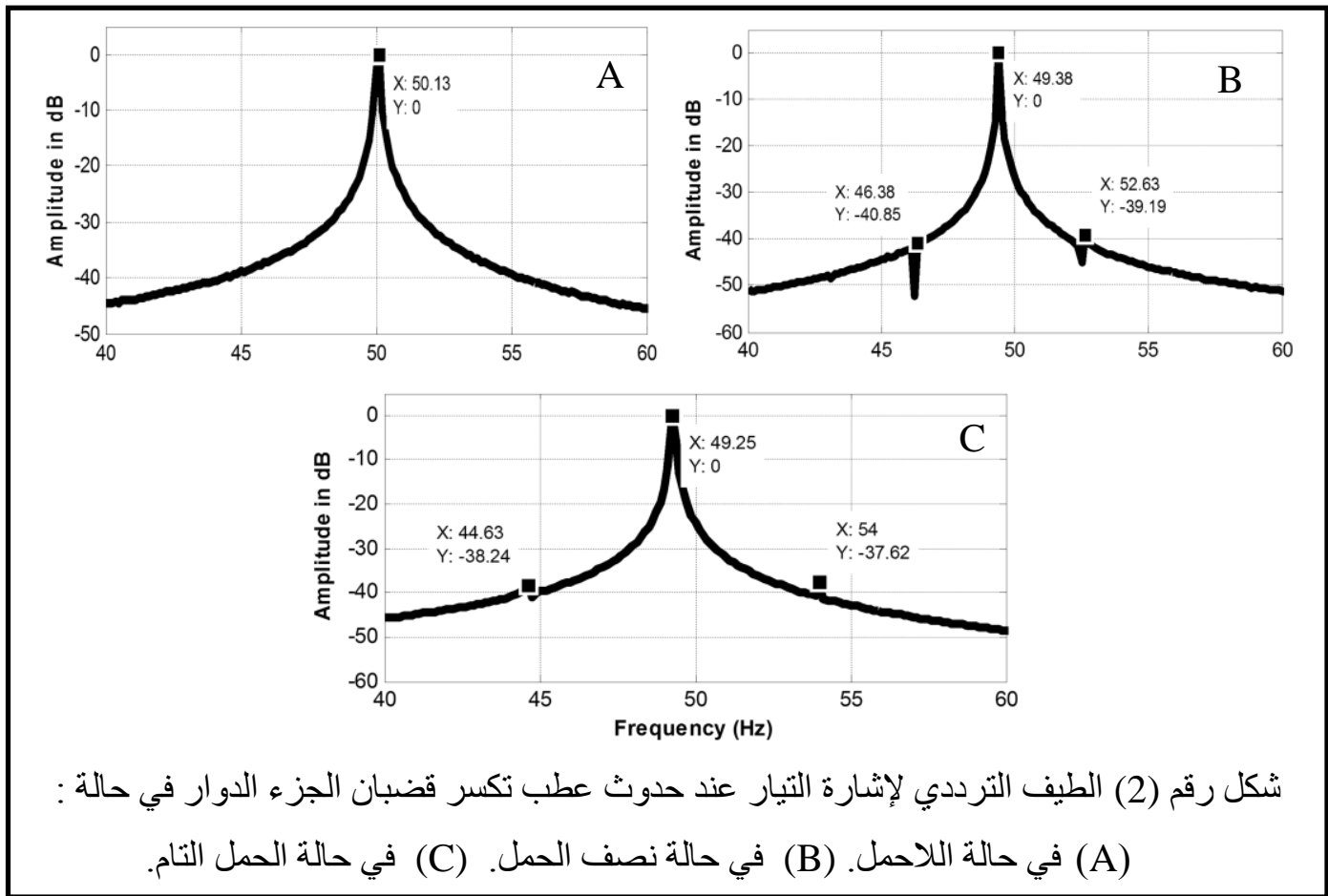

-2-2-4 تحليل إشارة الاهتزاز:-

تعتبر هذه الطريقة من الطرق الثنائعة في كثف الأعطاب الميكانيكية في مر احلها الأولية وتتم عن طريق تحليل

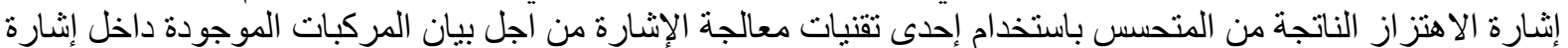

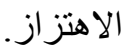

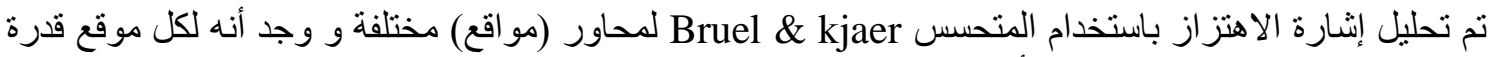

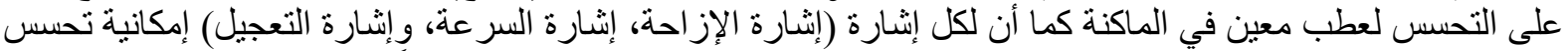

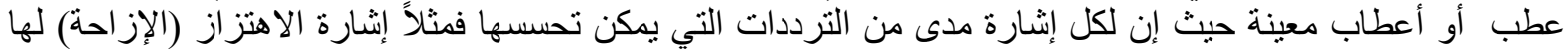

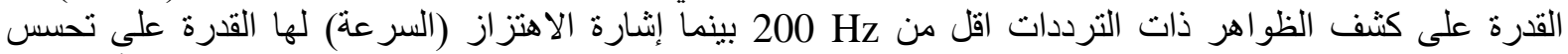

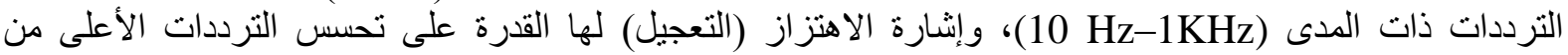

إن الجهاز Bruel \& Kjaer له القدرة على تزويدنا باشارة سر عة وتعجيل وخلال التحليل استنتجنا ما يلي:

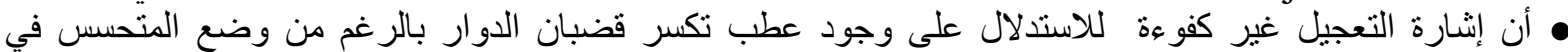
المحاور الثناثة.

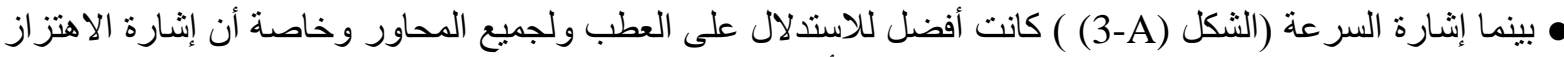

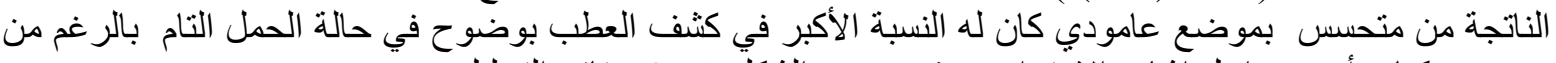

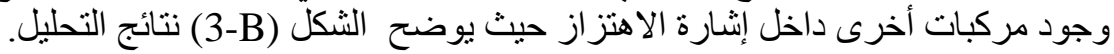

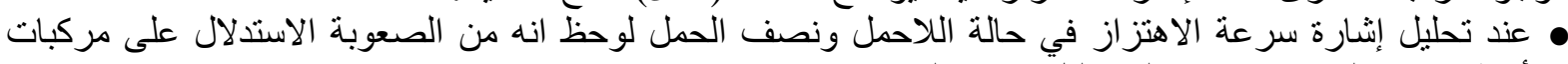

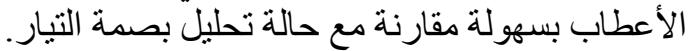

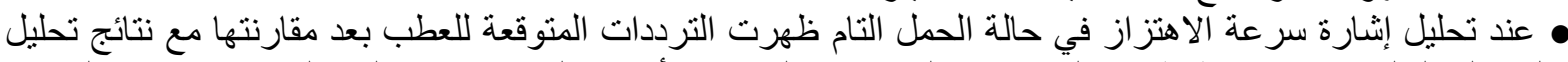
المحرك السليم حيث نلاحظ ظهور المركبات على جانبي المركبة الأساسية لتردد دوران المحرك. وكانت فئت قيمة المركبة 
الجانبية البسرى عند تردد (21.25 Hz) وبقيمة (38 (33.15 dB-) وكانت المركبة اليمنى عند تردد (25.88 ) وبقيمة

(3-B) (38.55 dB). (3) وكما موضح في النكل

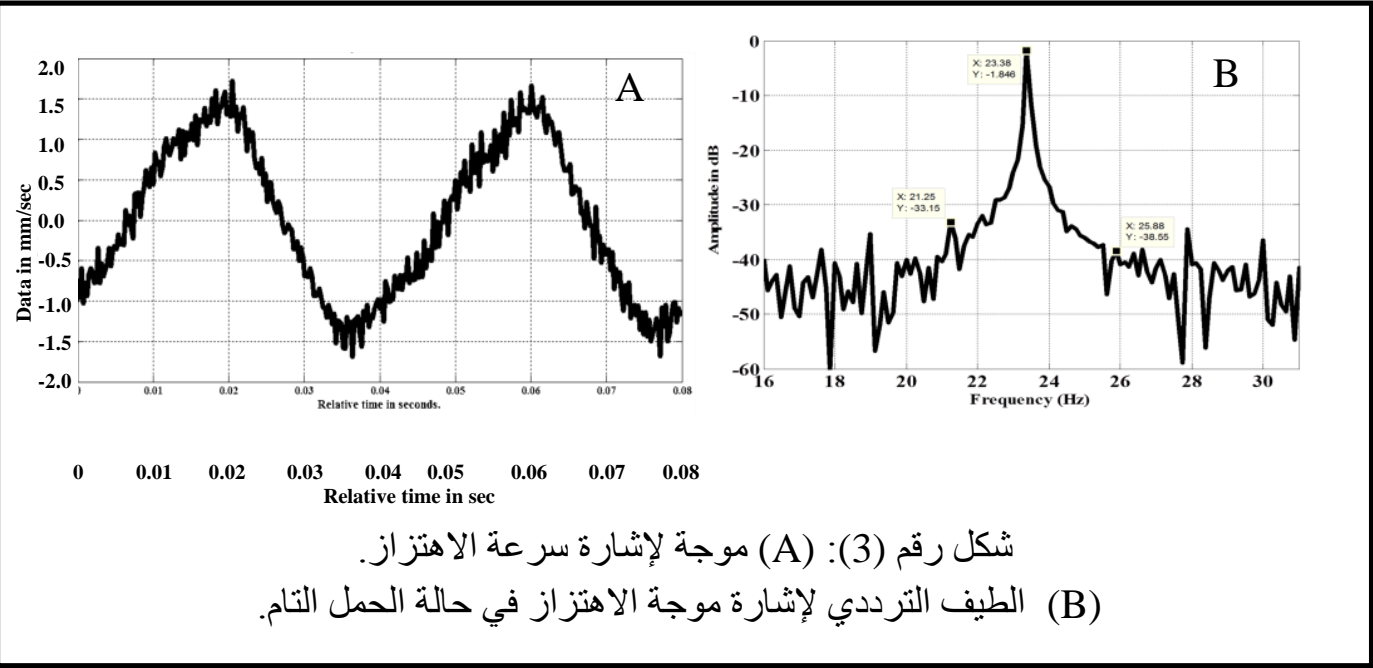

-3-2-4 تحليل الإشـارة الصوتية:-

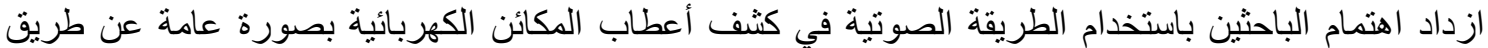

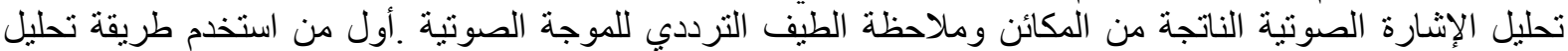

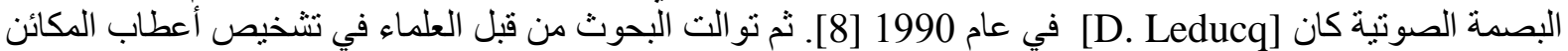

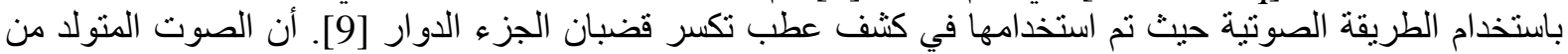

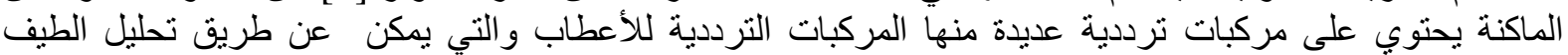

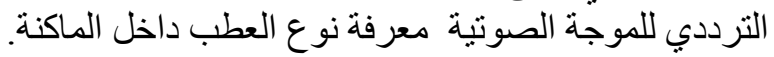

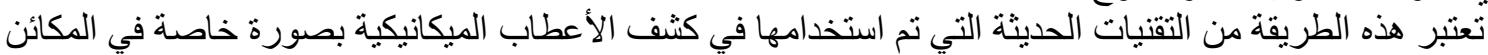

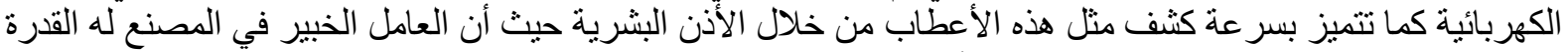

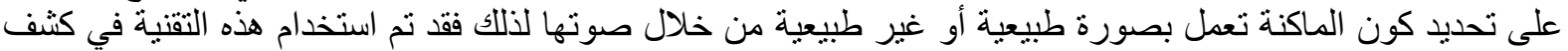

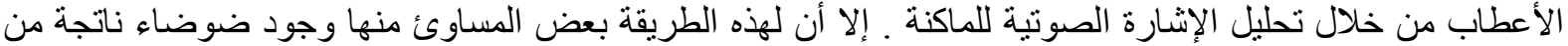

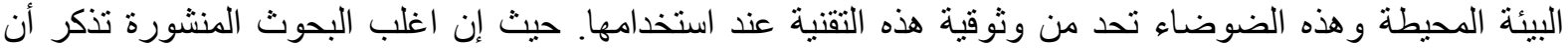

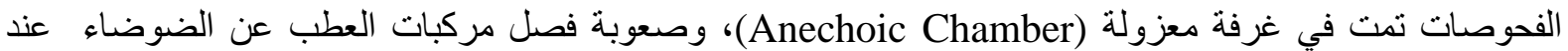

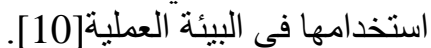

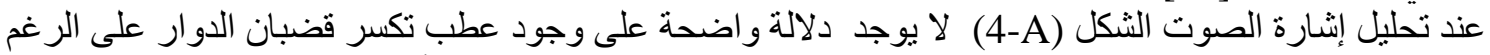

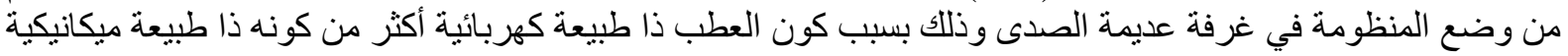

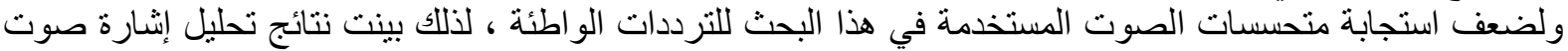

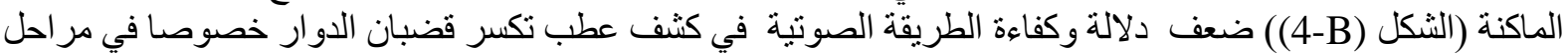
العطب المبكرة. رغم أن هذه الطريقة شخصت اعطاب المحامل واعطاب أخرى بكفاءة أفضل وبدلالة أوضح في بحوثنا السابقة [12][11]

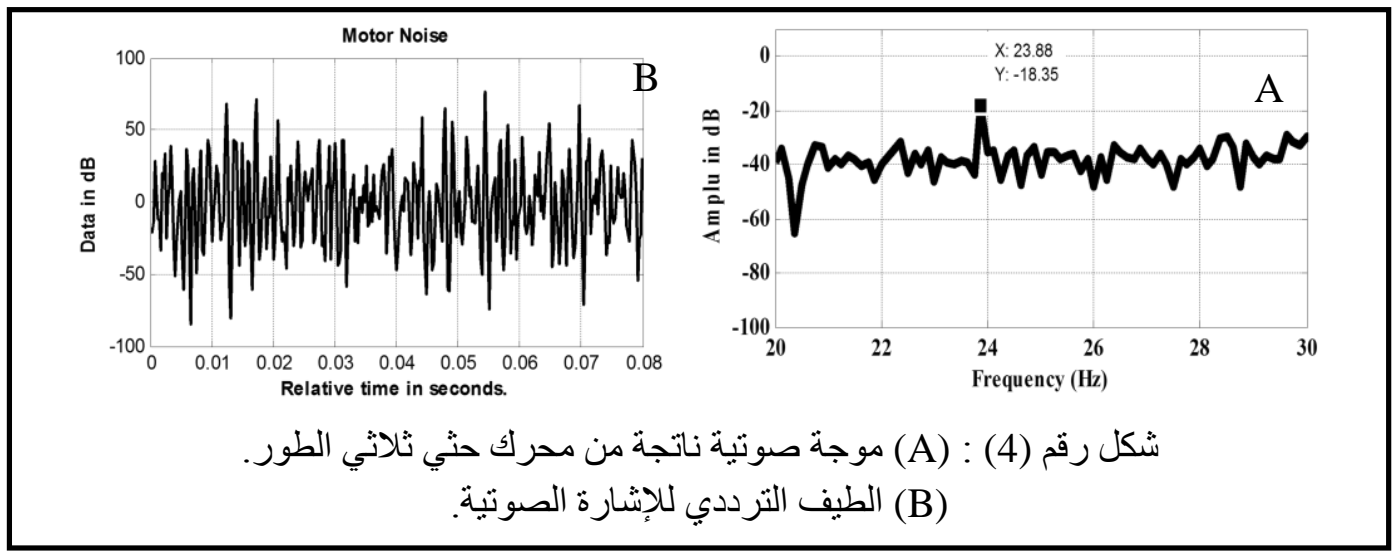


5. الاستنتاج

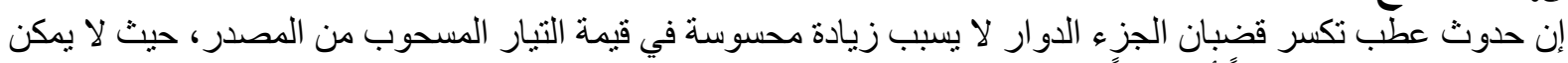

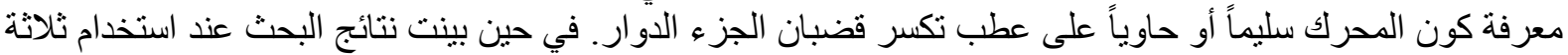

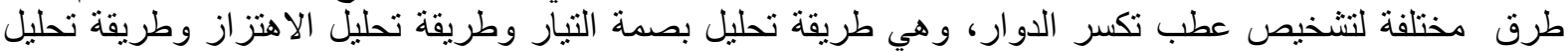

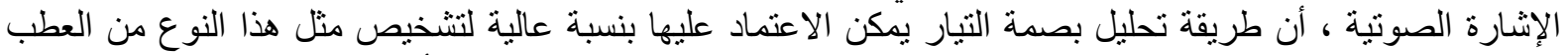

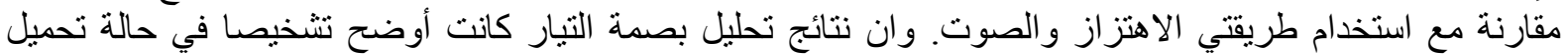

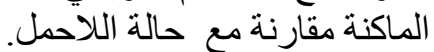

أما طريقة تحليل موجة الاهنز ازلة فقد أظهرت صعوبة في تمييز مركبات عطب تكسر قضبان الدوار في مر احله

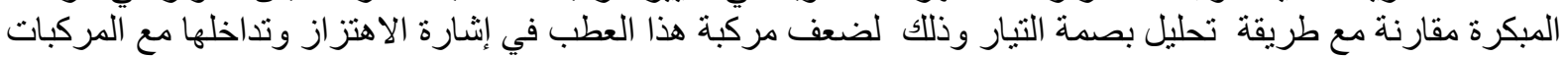

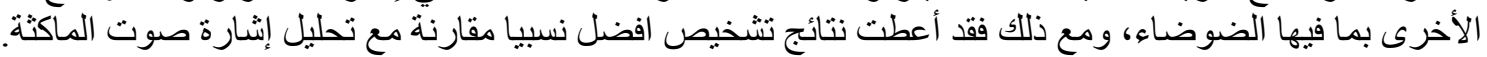

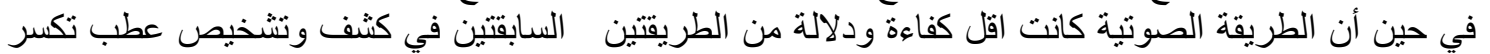

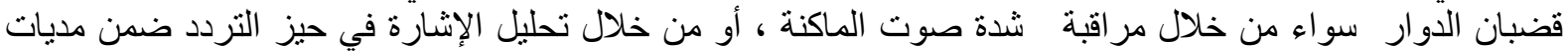

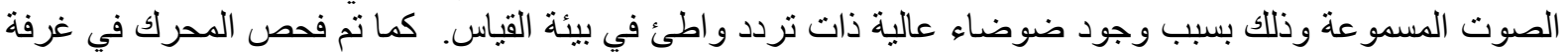

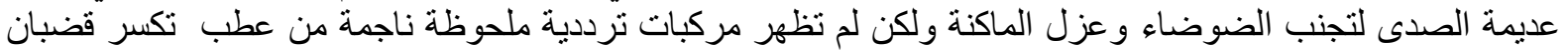

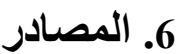

1. P. J. Tavner، "Review of Condition Monitoring of Rotating Electrical Machines"، IET Electrical power application، Vol. 2، No. 4، PP. (215-247)، 2008.

2. Yasir M. Y. Ameen، "Advanced Methods for Induction Motors Fault Diagnosis"، Ph. D. Thesis، Mosul University، Mosul، Iraq، 2007.

3. Clarence W. de Silva، "Vibration and Shock Handbook"، Mechanical Engineering Series، Frank Kreith - Series Editor، Chapter 25، 2005. (Book).

4. H. Douglas، P. Pillay، and A. K. Ziarani، "Broken Rotor Bar Detection in Induction Machines With Transient Operating Speeds"، IEEE Transactions on energy conversion، Vol. 20، No. 1، March 2005.

5. K. Abbasadeh، J. Milimonfared، M. Haji، H. A. Toliyat، "Detection of Broken rotor bars in induction motors using wavelet analysis"، IEEE industrial electronics society، 2001.

6. Subhasis Nandi ' Hamid A. Toliyat، and Xiaodong Li ‘" Condition Monitoring and Fault Diagnosis of Electrical Motors-A Review " ‘IEEE Transactions on energy conversion، Vol. 20، No. 4، December 2005

7. C. Kral، A. Haumer، and C. Grabner " Modeling and Simulation of Broken Rotor Bars in Squirrel Cage Induction Machines"، Proceedings of the World Congress on Engineering 2009، Vol. I، WCE 2009، July 1 - 3، 2009، London، U.K.

8. D. Leducq، Hydraulic noise diagnostics using wavelet analysis، Proceedings of the International Conference on Noise Control Engineering، 1990، pp. 997-1000.

9. Weidong Li، Chris K. Mechefske، "Detection of Induction Motor Faults: A Comparison of Stator Current، Vibration and Acoustic Methods"، Journal of Vibration and Control، 12(2): 165-188، 2006

10. J. R. Cameron، W. T. Thomson' and A. B. Dow" "Vibration and current monitoring for detecting air gap eccentricity in large induction motors،" Proceedings of IEE، Vol. 133، Pt. B، No. 3، May 1986، pp155-163.

11. M. H. Saud " Intelligent Methods for Three Phase Induction Motor Fault Detection and Diagnosis" Msc. Thesis, Mosul University, Mosul, Iraq, 2010.

12. B.M. Saied, Y.M.Y Ameen, M. H. Saud " Acostic Technique for Three-Phase Induction Motor Bearing Faults Detection" Proceedings of The Fifth Libyan Arab International Conference On Electrical and Electronic Engineering ,23-26 October, 2010.

$$
\text { تم اجر اء البحث في كلية ألهندة = جامعة ألموصل }
$$

